\title{
A New Route to Fabricate Multifunctional and Multistage Composite Nanoparticle
}

\author{
Xin Wang, Pin Chen, and Xiaohong Hu (D) \\ School of Material Engineering, Jinling Institute of Technology, Nanjing 211169, China \\ Correspondence should be addressed to Xiaohong Hu; hxh@jit.edu.cn
}

Received 16 March 2018; Revised 17 May 2018; Accepted 16 July 2018; Published 1 August 2018

Academic Editor: Ming-Guo Ma

Copyright (c) 2018 Xin Wang et al. This is an open access article distributed under the Creative Commons Attribution License, which permits unrestricted use, distribution, and reproduction in any medium, provided the original work is properly cited.

\begin{abstract}
The focus of nanoparticle research is on exploring its application in all kinds of field. Among these, multifunctional nanoparticle attracts increasing interest due to its fittest property and adjustable property. Herein, a multifunctional and multistage nanoparticle considering the advantage of both nanogel and $\mathrm{pH}$-responsive property has been designed and synthesized in the research. The composite nanoparticle was obtained by in situ processing and polymerization technique using acetylated $\beta$-cyclodextrin and gelatin as materials. Gelatin was first encapsulated into Ac- $\beta$-CD nanoparticle in order to investigate optimal fabrication conditions of $\mathrm{W} / \mathrm{O} / \mathrm{W}$ technique. The results showed that the nanoparticle had monodisperse characteristic and coarse spherical morphology, which was influenced by factors such as PVA concentration and water/oil ratio. In further step, two-phase composite nanoparticle could be obtained by combined W/O/W technique and in situ polymerization using optimal preparative parameters of $\mathrm{W} / \mathrm{O} / \mathrm{W}$ technique. Two-phase structure could be confirmed by TEM images and DLS results. Fabrication temperature had no effect on the diameters of composite nanoparticle, but influenced the encapsulated efficiency of nanogel. Finally, composite nanoparticle showed quick $\mathrm{pH}$ response property at mild acid medium and no obvious cytotoxicity.
\end{abstract}

\section{Introduction}

Nanoparticle is defined as a small sphere with nanoscale size, which attracts increasing interest due to its small-size effect in scientific research [1-3]. From one aspect, small-size effect brought not only large specific surface area but also flexible and adjustable properties [1-3]. From other aspect, nanoparticle is a bridge between bulk material and atomic structure, which offers a method to understand or establish the relationship between bulk material and atomic structure. To date, all kinds of metal, inorganic, and polymeric nanoparticles have been synthesized and prepared using gas condensation, attrition, chemical precipitation, microemulsion method, pyrolysis, and hydrothermal synthesis [2]. Among these kinds of nanoparticles, stimuli-responsive nanoparticles including $\mathrm{pH}$-sensitive, temperature-sensitive, enzymesensitive, photo-sensitive, and redox-sensitive have been intensively investigated in the application of biomedical, nanotechnology, information fields [4-9]. On account of mild acid environment in lesion locations and in endoso$\mathrm{mal} / \mathrm{lysosomal} \mathrm{compartments} \mathrm{of} \mathrm{cells,} \mathrm{low} \mathrm{pH}$-triggered nanoparticles have been used to deliver drugs, growth factors, and bioactive molecules into targeted tissue or cells [10-13]. Recently, quick response polysaccharide materials to mild acid environment had been synthesized and prepared to $\mathrm{pH}$-responsive nanofiber and nanoparticle $[10,14]$. Among these, $\mathrm{pH}$-sensitive acetylated $\beta$-cyclodextrin $(\mathrm{Ac}-\beta-\mathrm{CD}$ ) nanoparticle had been fabricated and introduced into injectable hydrogel [15]. The effectiveness and efficiency of Ac- $\beta$-CD nanoparticle had been confirmed by previous research [15].

However, nanoparticle with single function cannot satisfy multiaspect requirements like synergic delivery for two molecules with completely different properties, different response requirements, and different cell endocytosis properties in biomedical field. Since nanogel is another widely used nanoscale material with similar environment to physiological environment, it possesses a large number of advantages like good biocompatibility, similar mechanic properties to soft tissue, and less immune response $[16,17]$. On account of merits of both nanoparticle and nanogel, nanogel composite $\mathrm{pH}$-sensitive nanoparticles were designed using double 


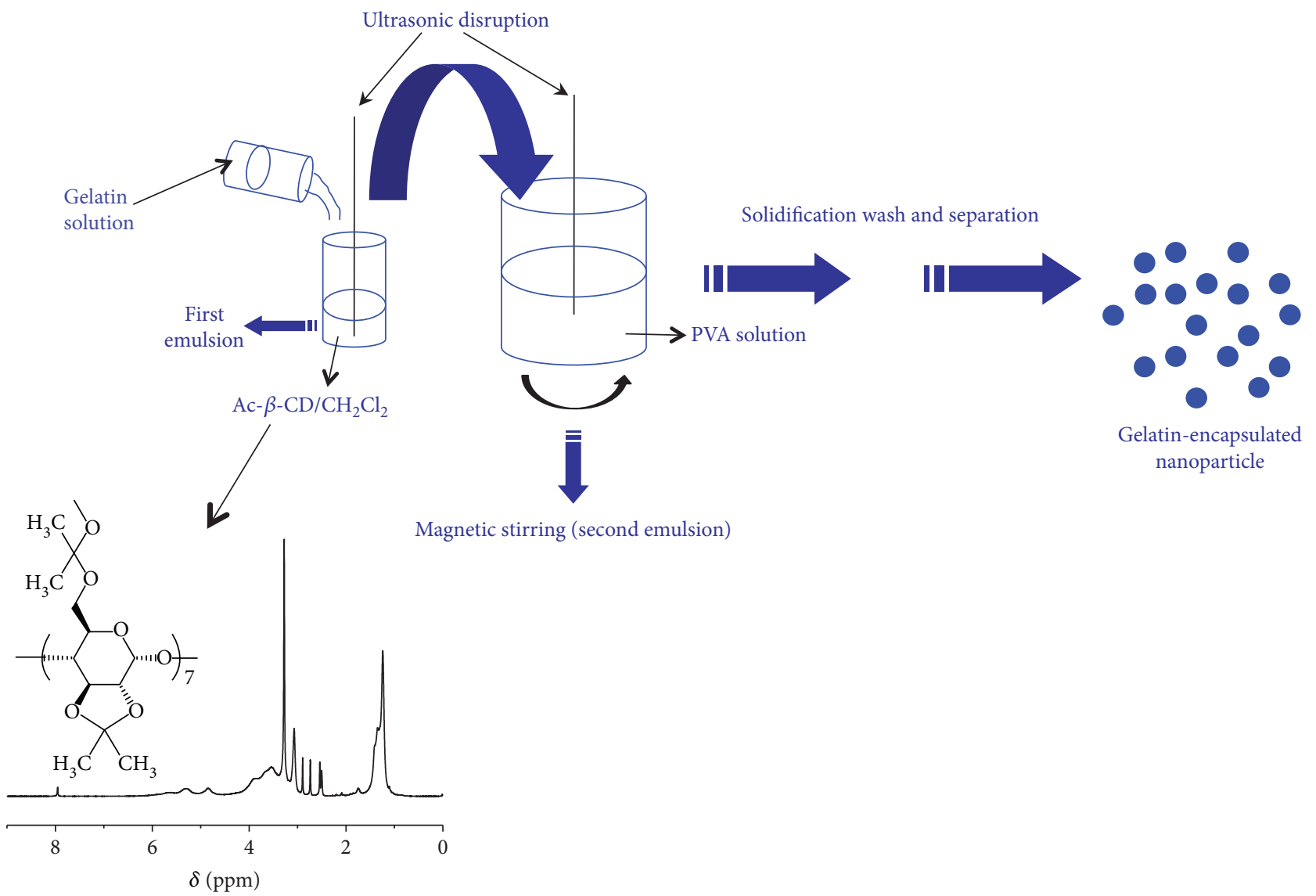

FIGURE 1: Schematic illustration to show the formation of gelatin-encapsulated nanoparticle.

emulsion method in the research. Since hydrogel is hydrophilic-crosslinked polymer network, hydrophilic natural polymer with crosslinkable points is an ideal material for nanogel synthesis. Combined with biocompatibility, biodegradability, and availability, crosslinkable gelatin, which was synthesized in our previous research, is a good choice for nanogel preparation. In order to explore the optimal conditions for double emulsion method, gelatin-encapsulated Ac- $\beta$-CD nanoparticles were first prepared and characterized. Then, two-phase composite $\mathrm{pH}$-sensitive nanoparticle was fabricated using similar method. Finally, structureproperty information of gelatin nanogel composite Ac- $\beta$ CD nanoparticle was investigated in the research. The aim of research is to provide a novel composite nanomaterial for future biomedical application.

\section{Experiment Section}

2.1. Materials. $\beta$-Cyclodextrin ( $\beta$-CD), 2-methoxypropene, pyridinium 4-toluenesulfonate, dimethyl sulfoxide (DMSO), dichloromethane (DCM), acrylic acid (AA, purified through vacuum distillation), gelatin, ammonium persulfate (APS), and $\mathrm{N}, \mathrm{N}, \mathrm{N}^{\prime}, \mathrm{N}^{\prime}$-tetramethylethylenediamine (TEMED) were purchased from Shanghai Chemical Industries Co. Ltd (China). Poly(vinyl alcohol) (PVA, 87-89 mol\% hydrolyzed, CPS: 4.6-5.4), 1-ethyl-3-(3-dimethylaminopropyl)carbodiimide hydrochloride (EDC), and N-hydroxysuccinimide
(NHS) were obtained from Aladdin (Shanghai) Co. Ltd. (China). Trypsin, Dulbecco's modified Eagle's medium (DMEM), and fetal calf serum (FBS) were purchased from Gibco. Fluorescein diacetate (FDA) and 3-(4,5-dimethyl) thiazol-2,5-dimethyl tetrazolium bromide (MTT) were got from Sigma. All other chemicals and reagents were used as received.

2.2. Synthesis of $G-A A$ and $A c-\beta-C D$. Acrylic acid-modified gelatin (G-AA) and acetylated $\beta$-cyclodextrin (Ac- $\beta$-CD) were synthesized according to previous reports [15]. Briefly, G-AA was obtained by EDC/NHS catalyzed coupling reaction and $A c-\beta-C D$ was synthesized by acetalization. In the protocol of G-AA synthesis, carboxyl group was first activated by EDC/NHS with molar ratio of $1: 1.2: 0.5$ ( $\mathrm{pH} 4.0-4.5$ ) for $30 \mathrm{~min}$, then activated AA was reacted with gelatin in water solution ( $\mathrm{pH} 5.0-5.5)$ for $10 \mathrm{~h}$ at room temperature. Finally, G-AA was obtained by dialyzing and freeze-drying. In the protocol of Ac- $\beta$-CD synthesis, $50 \mathrm{mM}$ $\beta$-CD was reacted with 2 -methoxypropene with molar ratio of 1 : 40 under catalysis of $5 \mathrm{mM}$ pyridinium p-toluene sulfonate in anhydrous anaerobic DMSO environment at $30^{\circ} \mathrm{C}$ for $1 \mathrm{~h}$. Then, Ac- $\beta$-CD was obtained by precipitation and filtration and lyophilized. The structure of Ac- $\beta$-CD was characterized by ${ }_{1} \mathrm{H}$ NMR (Figure 1 ), which was assigned as follows: the chemical shifts from $1.0 \mathrm{ppm}$ to $2.0 \mathrm{ppm}$ are attributed to the protons of $\mathrm{CH}_{3}-\mathrm{C}$ at 1 and $1^{\prime}$ positions, the 


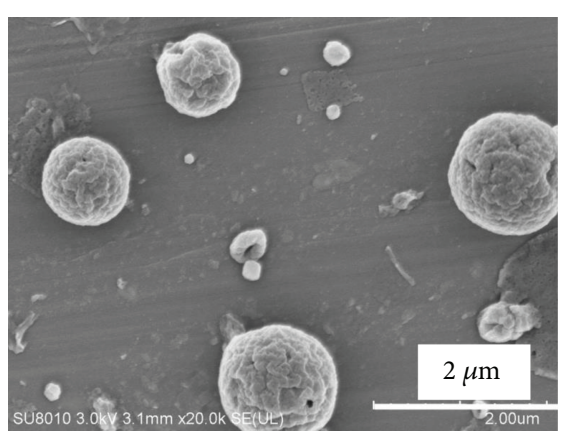

(a)

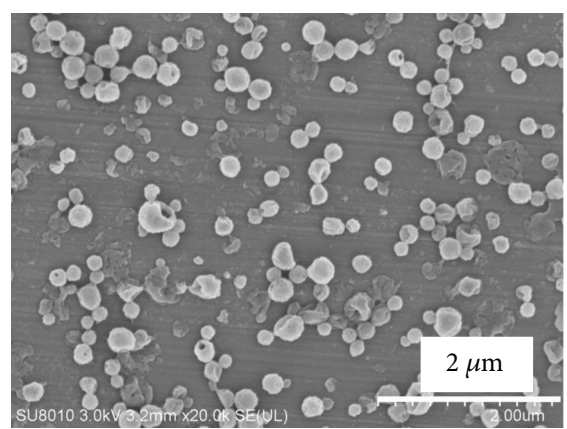

(b)

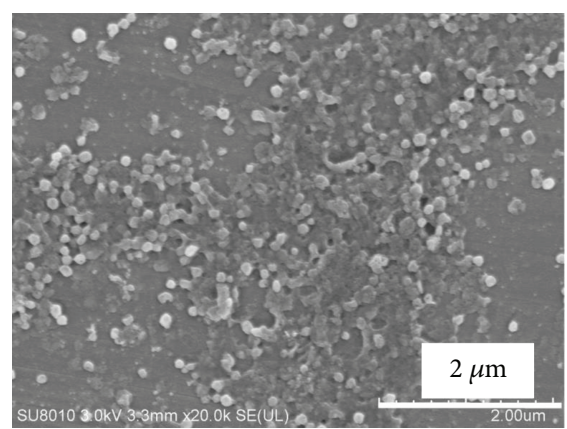

(c)

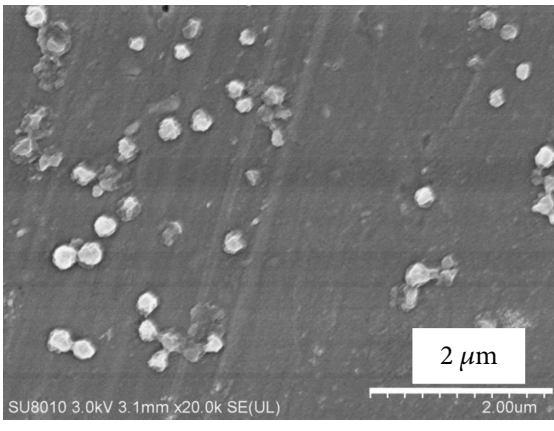

(d)

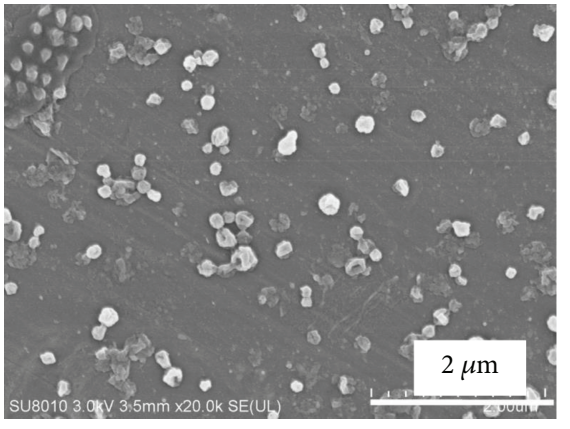

(e)

FIgURe 2: SEM images of Nano1 (a), Nano2 (b), Nano3 (c), Nano4 (d), and Nano5 (e).

chemical shift at $3.0 \mathrm{ppm}$ is attributed to the protons of $\mathrm{CH}_{3}-\mathrm{O}$ at 2 position, the chemical shifts from $3.2 \mathrm{ppm}$ to $4.0 \mathrm{ppm}$ are attributed to the protons of $\beta$-CD at 3,4 , and 5 positions, respectively, and the chemical shifts at $4.6 \mathrm{ppm}$ and $5.4 \mathrm{ppm}$ are attributed to the protons of 6 position [14].

\subsection{Preparation and Characterization of Gelatin-} Encapsulated Nanoparticle. Gelatin-encapsulated nanoparticles were prepared by double microemulsion method. Briefly, $200 \mu \mathrm{L}$ of $10 \%$ gelatin solution was first emulsified via probe sonication (Scientz, JY92-II) into $1 \mathrm{~mL}$ of $10 \% \mathrm{w} / v$ Ac- $\beta$-CD/DCM solution, which was further emulsified into $6 \mathrm{~mL}$ of $1 \% w / v$ PVA aqueous solution. The obtained emulsion was immediately added into $20 \mathrm{~mL}$ of $0.3 \% w / v$ PVA solution to evaporate DCM under magnetic stirring. Finally, nanoparticles were collected by centrifugation $(14,000 \mathrm{rpm}$, $10 \mathrm{~min}$ ) after $10 \mathrm{~h}$. The collected nanoparticles were washed several times with basic water, lyophilized, and characterized by dynamic light scattering (DLS, nano ZS), scanning electron microscope (SEM, S8100). For DLS test, intensity average was been given to evaluate nanoparticle size. The gelatin encapsulated in nanoparticle was determined by BCA assay. Briefly, $20 \mu \mathrm{L}$ of dissolved nanoparticle solution was added to one well of a 96-well culture plate, into which $200 \mu \mathrm{L} \mathrm{BCA}$ (agent $\mathrm{A} /$ agent $B=50 / 1$ ) solution was added. After $30 \mathrm{~min}$ at $60^{\circ} \mathrm{C}$, the absorbance at $570 \mathrm{~nm}$ was recorded on a microplate reader (Tecan M200), which was used to calculate the gelation concentration by referring to a calibration curve. Encapsulation efficiency of gelatin in nanoparticle was calculated by encapsulated gelatin amount* $100 \%$ /original gelatin amount.
2.4. Fabrication and Characterization of Two-Phase Composite Nanoparticle. Two-phase composite nanoparticles were also fabricated by double microemulsion method. Briefly, G-AA was dissolved in PBS to obtain $10 \% w / v$ G-AA solution, into which $50 \mathrm{mM}$ APS was added to form homogeneous solution. $200 \mu \mathrm{L}$ above solution was emulsified into $1 \mathrm{~mL}$ of $10 \% w / v$ Ac- $\beta$-CD/DCM solution, which was further emulsified into $6 \mathrm{~mL}$ of $1 \% \mathrm{w} / v$ PVA aqueous solution containing $50 \mathrm{mM}$ TEMED. The obtained emulsion was immediately added into $20 \mathrm{~mL}$ of $0.3 \% w / v$ PVA solution to evaporate DCM under magnetic stirring at different temperatures. At the same time, G-AA was crosslinked by radical polymerization to form nanogel structure. Finally, nanoparticles were collected by centrifugation $(14,000 \mathrm{rpm}, 10 \mathrm{~min})$ after $10 \mathrm{~h}$. The collected nanoparticles were washed several times with basic water, lyophilized, and characterized by dynamic light scattering (DLS, nano ZS), scanning electron microscope (SEM, S8100), and transmission electron microscope (TEM, Tecnai 12) at $200 \mathrm{kV}$. For DLS test, intensity average was been given to evaluate nanoparticle size. Moreover, transparency at $400 \mathrm{~nm}$ for nanoparticle solution in different mediums as a function of time was detected by UV spectroscopy (Cary 50). The gelatin encapsulated in nanoparticle was also determined by the abovementioned BCA assay.

In vitro cultured MCF-7 cells (95\% humidified atmosphere $+5 \% \mathrm{CO}_{2}, 37^{\circ} \mathrm{C}$ ) were used to preliminarily evaluate the cytotoxicity of two-phase composite nanoparticle, which were detached using $0.25 \%$ trypsin in PBS before use. In the protocol of cytotoxicity evaluation, $100 \mu \mathrm{L}$ of $200 \mu \mathrm{g} / \mathrm{mL}$ nanoparticle DMEM suspension was added into each well 
TABLE 1: Fabrication condition and characterizations of gelatin-encapsulated nanoparticles.

\begin{tabular}{lccccccc}
\hline & \multicolumn{2}{c}{ Preparative parameters } & \multicolumn{4}{c}{ Characterization } \\
Entry & $\begin{array}{c}\text { PVA conc. } \\
(w / v, \%)\end{array}$ & $\begin{array}{c}\text { Water/oil ratio of the } \\
\text { first emulsion }\end{array}$ & $\begin{array}{c}\text { Diameter } \\
(\mathrm{nm}, \mathrm{SEM})\end{array}$ & $\begin{array}{c}\text { Hydrodynamic diameter } \\
(\mathrm{DLS})\end{array}$ & $\begin{array}{c}\text { Zeta potential } \\
(\mathrm{mV})\end{array}$ & $\begin{array}{c}\text { Encapsulation } \\
\text { Efficiency }(\%)\end{array}$ \\
\hline Nano1 & 1 & $1: 5$ & $324 \pm 180$ & 313 & 0.091 & $-10.7 \pm 4.6$ & $1.8 \pm 0.3$ \\
Nano2 & 3 & $1: 5$ & $194 \pm 60$ & 253 & 0.150 & $-12.1 \pm 7.3$ & $1.1 \pm 0.1$ \\
Nano3 & 5 & $1: 5$ & $98 \pm 14$ & 191 & 0.092 & $-9.3 \pm 11.5$ & $1.4 \pm 0.4$ \\
Nano4 & 3 & $1: 10$ & $179 \pm 58$ & 266 & 0.111 & $-14.0 \pm 9.4$ & $1.1 \pm 0.1$ \\
Nano5 & 3 & $1: 20$ & $150 \pm 63$ & 267 & 0.227 & $-12.0 \pm 8.1$ & $1.0 \pm 0.1$ \\
\hline
\end{tabular}

TABLE 2: Fabrication condition and characterizations of biphase nanoparticles.

\begin{tabular}{lcccccc}
\hline & & \multicolumn{2}{c}{ Hydrodynamic diameter } \\
Entry & Preparation temperature $\left({ }^{\circ} \mathrm{C}\right)$ & \multicolumn{2}{c}{ In PBS } & \multicolumn{2}{c}{ In acid } & Encapsulation efficiency (\%) \\
& & 199 & 0.316 & 245 & 0.191 & $12.0 \pm 3.1$ \\
\hline Nano20 & 20 & Eff. diam. $(\mathrm{nm})$ & PDI & Eff. diam. (nm) & PDI \\
Nano40 & 40 & 195 & 0.297 & 253 & 0.150 & $14.1 \pm 2.9$ \\
\hline
\end{tabular}

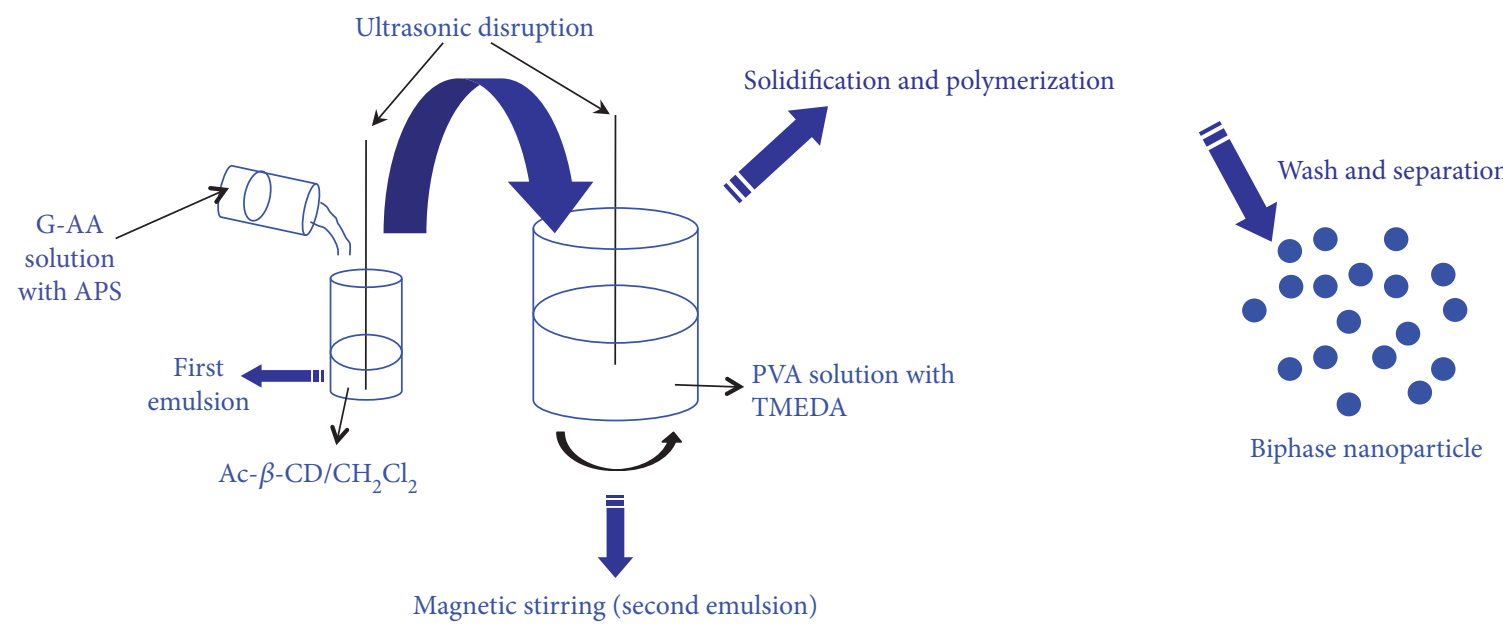

FIGURE 3: Schematic illustration to show the formation of biphase nanoparticle.

of 96-well culture plate, into which $100 \mu \mathrm{L}$ cell suspension containing 2000 cells was subsequently added. After $24 \mathrm{~h}$, MTT assay and cell images were used to characterize cytoviability. In MTT assay, $20 \mu \mathrm{L}$ MTT was incubated with live cells in each well for $4 \mathrm{~h}$ to form formazan pigment, which was further dissolved by $200 \mu \mathrm{L}$ DMSO. Cytoviability was estimated according to the relative absorbance at $490 \mathrm{~nm}$ by a microplate reader (Multiskan FC). In addition, viable cells were stained by $5 \mu \mathrm{g} / \mathrm{mL}$ FDA for $10 \mathrm{~min}$, washed with PBS for several times, and observed by fluorescence microscopy (IX73).

2.5. Statistical Analysis. Data were analyzed using the $t$-test for differences. Results are reported as means \pm standard deviation. The significant level was set at $p<0.05$.

\section{Results and Discussion}

3.1. Gelatin-Encapsulated Nanoparticle. The detailed structural information of Ac- $\beta-C D$ and G-AA was described in our previous research $[14,15]$. Gelatin-encapsulated Ac- $\beta$-CD nanoparticle was fabricated by double water-inoil-in-water $(\mathrm{W} / \mathrm{O} / \mathrm{W})$ technique, which was illustrated in Figure 1. Detailedly, gelatin aqueous solution was encapsulated into Ac- $\beta$-CD/DCM solution to form the first emulsion, which was further emulsified into water with emulsifier (PVA) to form nanoparticle after DCM was evaporated. Finally, gelatin-encapsulated Ac- $\beta$-CD nanoparticle was obtained by wash and separation. Since the influence of preparation conditions on Ac- $\beta$-CD nanoparticle's (single O/W technique) structure property had been investigated previously, the optimal preparation conditions like $10 \%$ Ac- $\beta$-CD 


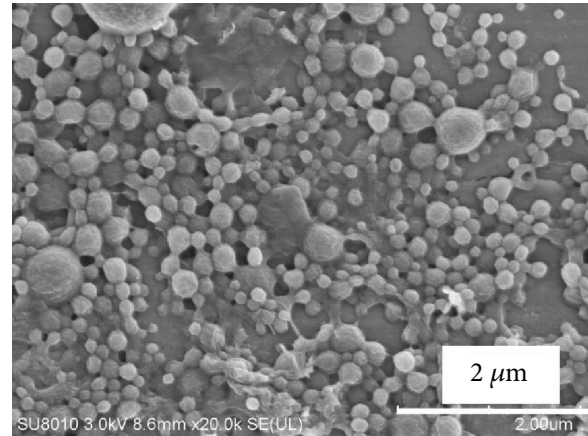

(a)

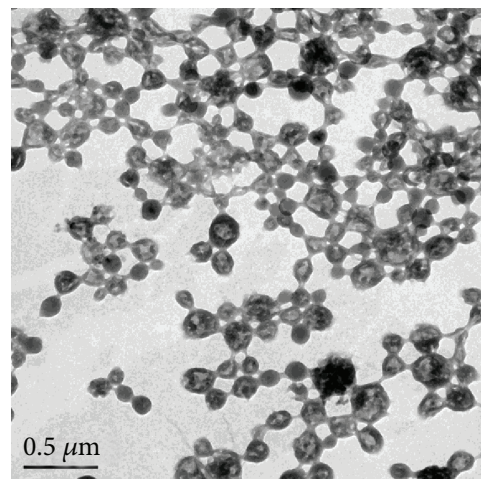

(c)

Figure 4: SEM images of Nano20 (a) and Nano40 (b). TEM images of Nano20 (c) and Nano40 (d).

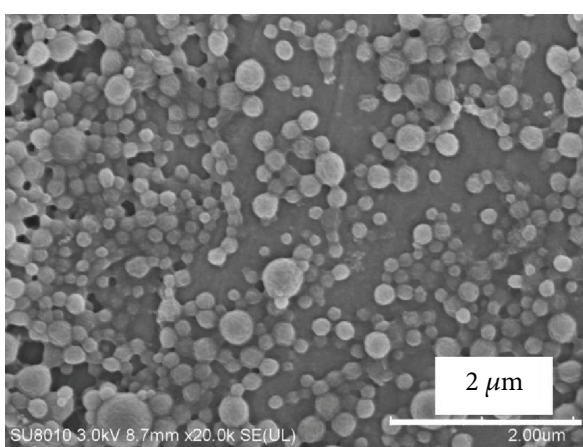

(b)

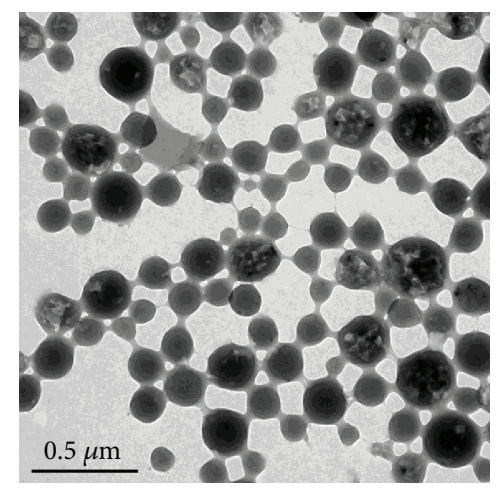

(d) solution and $1: 6 \mathrm{oil} /$ water ratios for the second emulsion were chosen and fixed in the research. Moreover, preparative parameters like PVA concentration and water/oil ratio of the first emulsion were studied in Figure 2 and Table 1 in the work. All formed nanoparticles exhibited monodisperse characteristic, nearly spherical morphology, and coarse surface, confirmed by DLS results and SEM image (Table 1 and Figure 2). Detailedly, either statistic diameter from SEM images or effective diameter from DLS results decreased with the increase of PVA concentration. However, nanoparticle showed not regular sphere with many defects with higher PVA concentration (Figure 2(c)) and lower water/oil ratio (Figures 2(d) and 2(e)). Additionally, when PVA concentration was higher, nanoparticles seemed to stick together (Figure 2(c)), which might be attributed to unwashed PVA. The water/oil ratio of the first emulsion showed little effect on the diameter of nanoparticle either from SEM images or from DLS results. Moreover, zeta potential of all nanoparticles was from $-9.3 \mathrm{mV}$ to $-14.0 \mathrm{mV}$ (Table 2). No significant difference was detected for zeta potential of nanoparticle for different preparative parameters. Encapsulation efficiency of inner material is another index to characterize effectiveness for functional nanoparticle. BCA results showed that gelatin could be encapsulated into nanoparticle using this method. But unfortunately, gelatin encapsulation efficiency was as low as $1.0-2.0 \% w / v$. When PVA concentration was low in preparation, gelatin encapsulation efficiency was higher than other nanoparticles, which might be due to less wash time in preparation. These results indicated that gelatin inside nanoparticle could diffuse into outside aqueous solution.

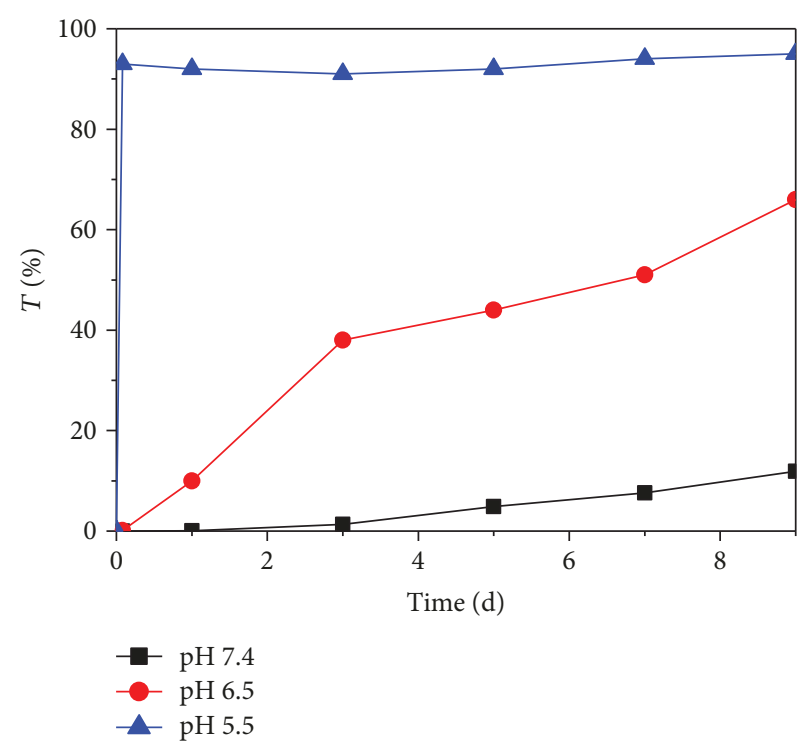

FIgURE 5: Transparency at $400 \mathrm{~nm}$ of biphase nanoparticle solutions with different $\mathrm{pH}$ values.

3.2. Two-Phase Composite Nanoparticle. As far as nanoparticle size, morphology, and probable emulgator remaining from abovementioned results, preparative parameters of $\mathrm{Nano} 2$ as an optimal condition for $\mathrm{W} / \mathrm{O} / \mathrm{W}$ technique were chosen for further investigation. Since crosslinkable gelatin had been verified to be an effective material for hydrogel 


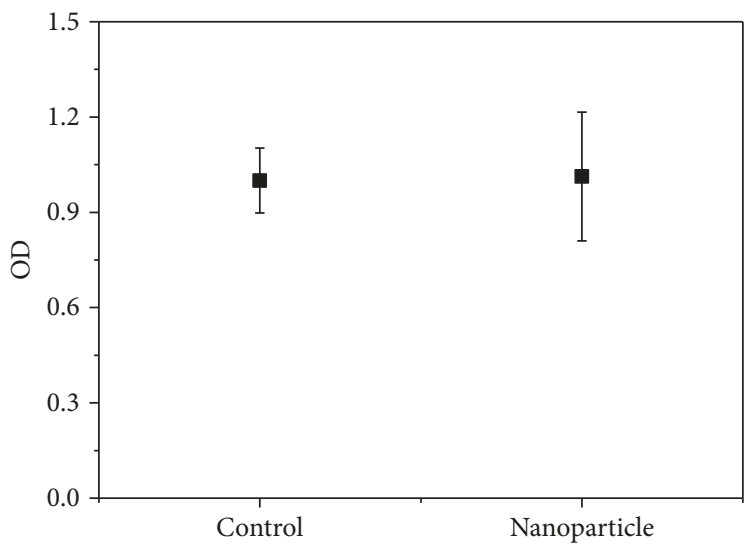

(a)

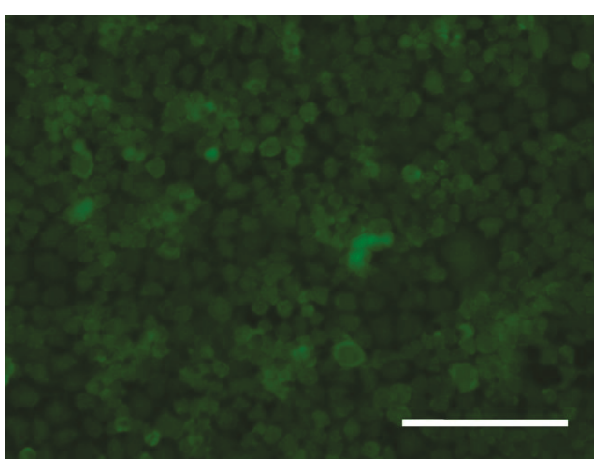

(b)

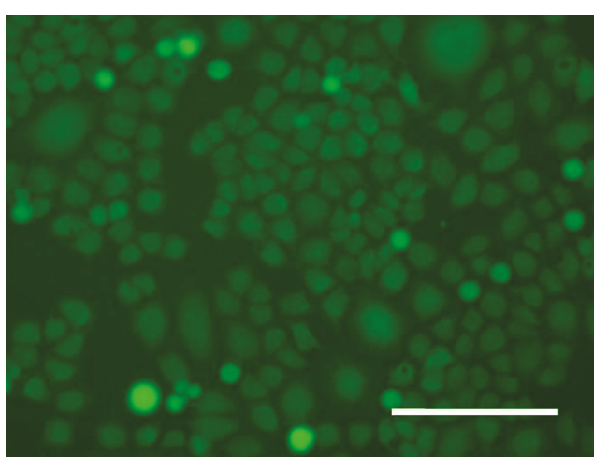

(c)

Figure 6: (a) Cytoviability of MCF-7 cells control and cocultured with Nano40. Stained MCF-7 cells observed by fluorescence microscopy after cultured $1 \mathrm{~d}$ (b, c). MCF-7 cells control (b). Cells cocultured with $100 \mu \mathrm{g} / \mathrm{mL}$ Nano40 (c). The scale is $200 \mu \mathrm{m}$.

fabrication with functional properties, it was encapsulated in nanoparticle for in situ nanogel fabrication just as illustrated in Figure 3. In order to make gelatin crosslinked, redox initiation system (APS/TEMED) was used and dissolved in the first and second emulsions. During the process of nanoparticle fabrication, in situ polymerization was taken place simultaneously. Finally, uniformly distributed nanoparticles were obtained regardless of fabrication temperature, which was confirmed by SEM images (Figures 4(a) and 4(b)) and TEM images (Figures 4(c) and 4(d)). In particular, biphase structure was clearly seen in TEM image, which indicated that nanogel embedded in nanoparticle had been successfully formed. Moreover, fabrication temperature seemed to have little influence on the size and morphology of nanoparticles either from SEM images or from TEM images. Hydrodynamic diameters of composite nanoparticle in PBS and in acid were detected by DLS, respectively, which was shown in Table 2. Since Ac- $\beta$-CD could be dissolved in acid solution according to previous research, diameter in acid was considered to be the diameter of inner nanogel due to degradation of Ac- $\beta$-CD. It was found that the effective diameter of composite nanoparticle was around $200 \mathrm{~nm}$ and the effective diameter of nanogel was around $250 \mathrm{~nm}$ regardless of fabrication temperature. Moreover, the diameter of nanogel is a little bigger than that of composite nanoparticle, which might be ascribed to the extension of hydrophilic nanogel network in water without hydrophobic Ac- $\beta$-CD outsider layer. Furthermore, encapsulated efficiency of nanogel was about $12-14 \% w / v$, which was much larger than that of abovementioned uncrosslinked gelatin. In addition, encapsulated efficiency of nanogel for Nano40 is a little higher than that for Nano20 for the reason that higher fabrication temperature resulted in fast polymerization rate to hinder gelatin molecule diffusion.

Transparency of nanoparticle solution was used to track the degradation process of nanoparticle based on the fact that solution became clear after Ac- $\beta$-CD was degraded and dissolved in solution (Figure 5). In PBS ( $\mathrm{pH} 7.4$ ), transparency of nanoparticle solution at $400 \mathrm{~nm}$ nearly kept unchanged during the first $3 \mathrm{~d}$, then slowly increased up to $10 \%$ in the following $6 \mathrm{~d}$, indicating relative stability of nanoparticle in that solution. In pure water ( $\mathrm{pH} 6.5)$, transparency of nanoparticle solution at $400 \mathrm{~nm}$ gradually increased up to $60 \%$ with prolonged time, indicating the continuous degradation of nanoparticle in $9 \mathrm{~d}$. In mild acid solution ( $\mathrm{pH} 5.5$ ), transparency of nanoparticle solution at $400 \mathrm{~nm}$ rapidly increased to $90 \%$ in the first day, indicating quickly degradation of nanoparticle. The results confirmed the quick $\mathrm{pH}$ response property at mild acid medium. The detailed degradation mechanism had been discussed in our previous research. Briefly, degradation of Ac- $\beta$-CD nanoparticle was due to structural change of Ac- $\beta$-CD. Synthesized Ac- $\beta-C D$ 
containing linear acetal groups (LA) and cyclic acetal groups (CA) is sensitive to low $\mathrm{pH}$ environment, which was confirmed by ${ }_{1} \mathrm{H}$ NMR spectrum.

In view of abovementioned properties, Nano40 was chosen for further cytotoxicity evaluation. Nano40 was cocultured with MCF-7 cells to evaluate its preliminary biocompatibility in vitro. As shown in Figure 6(a), the viability of cells cultured with Nano40 was a little higher than TCPs control, but without significant difference. Fluorescence microscopy images confirmed that live cells lived on the plate homogeneously (Figures 6(b) and 6(c)), with or without Nano40. These results confirmed that the Nano40 nanoparticle could support cell survival.

\section{Conclusion}

Gelatin-encapsulated Ac- $\beta$-CD nanoparticle with monodisperse characteristic, nearly spherical morphology, and coarse surface could be easily fabricated by double water-in-oil-inwater $(\mathrm{W} / \mathrm{O} / \mathrm{W})$ technique. The diameters of nanoparticles decreased with increase of PVA concentration, but were not influenced by water/oil ratio of the first emulsion. But nanoparticle showed sticky and irregular sphere shape with many defects when PVA concentration was higher and water/oil ratio was lower. Zeta potential of the nanoparticles was from $-9.3 \mathrm{mV}$ to $-14.0 \mathrm{mV}$. Further two-phase composite nanoparticle could be obtained by combined $\mathrm{W} / \mathrm{O} / \mathrm{W}$ technique and in situ polymerization. Two-phase structure could be confirmed by TEM images. The effective diameter of composite nanoparticle was around $200 \mathrm{~nm}$, and the effective diameter of nanogel was around $250 \mathrm{~nm}$ regardless of fabrication temperature. Encapsulated efficiency of nanogel was about $12-14 \%$, and higher fabrication temperature resulted in higher encapsulated efficiency. Composite nanoparticle showed quick $\mathrm{pH}$ response property at mild acid medium and no obvious cytotoxicity.

\section{Data Availability}

All the data supporting the results are shown in the article and can be available from the corresponding author.

\section{Conflicts of Interest}

The authors declare that there are no competing interests regarding the publication of this paper.

\section{Acknowledgments}

This study is financially supported by Natural Science Foundation of Jiangsu Province (BK20171113), Qing Lan Project, the Scholarship of Jiangshu Government, Six Talent Peaks Project in Jiangsu Province (JY-071).

\section{References}

[1] A. L. Armstead and B. Li, "Nanotoxicity: emerging concerns regarding nanomaterial safety and occupational hard metal (WC-Co) nanoparticle exposure," International Journal of Nanomedicine, vol. 11, pp. 6421-6433, 2016.
[2] D. Bobo, K. J. Robinson, J. Islam, K. J. Thurecht, and S. R. Corrie, "Nanoparticle-based medicines: a review of FDAapproved materials and clinical trials to date," Pharmaceutical Research, vol. 33, no. 10, pp. 2373-2387, 2016.

[3] H. Wang, J. Yu, X. Lu, and X. He, Nanomedicine, vol. 11, no. 2, pp. 103-106, 2016.

[4] M. R. Preiss and G. D. Bothun, "Stimuli-responsive liposomenanoparticle assemblies," Expert Opinion on Drug Delivery, vol. 8, no. 8, pp. 1025-1040, 2011.

[5] A. R. Town, M. Giardiello, R. Gurjar et al., Nanoscale, vol. 9, no. 19, pp. 6302-6314, 2017.

[6] Y. Zhao, K. Thorkelsson, A. J. Mastroianni et al., "Smallmolecule-directed nanoparticle assembly towards stimuliresponsive nanocomposites," Nature Materials, vol. 8, no. 12, pp. 979-985, 2009.

[7] J. Dong, Y. Wang, J. Zhang et al., "Multiple stimuli-responsive polymeric micelles for controlled release," Soft Matter, vol. 9, no. 2, pp. 370-373, 2013.

[8] S. Chen, F. Jiang, Z. Cao, G. Wang, and Z.-M. Dang, "Photo, $\mathrm{pH}$, and thermo triple-responsive spiropyran-based copolymer nanoparticles for controlled release," Chemical Communications, vol. 51, no. 63, pp. 12633-12636, 2015.

[9] Z. Cao, H. Wu, J. Dong, and G. Wang, "Quadruple-stimulisensitive polymeric nanocarriers for controlled release under combined stimulation," Macromolecules, vol. 47, no. 24, pp. 8777-8783, 2014.

[10] E. M. Bachelder, E. N. Pino, and K. M. Ainslie, "Acetalated dextran: a tunable and acid-labile biopolymer with facile synthesis and a range of applications," Chemical Reviews, vol. 117, no. 3, pp. 1915-1926, 2017.

[11] B. Wang, P. Liu, B. Shi, J. Gao, and P. Gong, "Preparation of $\mathrm{pH}$-sensitive dextran nanoparticle for doxorubicin delivery," Journal of Nanoscience and Nanotechnology, vol. 15, no. 4, pp. 2613-2618, 2015.

[12] Y. Wang, H. Chen, Y. Liu et al., Biomaterials, vol. 34, no. 29, pp. 7181-7190, 2013.

[13] Z. Zare-Akbari, H. Farhadnejad, B. Furughi-Nia, S. Abedin, M. Yadollahi, and M. Khorsand-Ghayeni, "PH-sensitive bionanocomposite hydrogel beads based on carboxymethyl cellulose/ZnO nanoparticle as drug carrier," International Journal of Biological Macromolecules, vol. 93, no. Part A, pp. 1317-1327, 2016.

[14] X. Hu, S. Chen, X. Gong, Z. Gao, X. Wang, and P. Chen, "Synthesis and preparation of biocompatible and $\mathrm{pH}$-responsive cyclodextrin-based nanoparticle," Journal of Nanoparticle Research, vol. 19, no. 3, p. 109, 2017.

[15] B. Chen and X. Hu, "An injectable composite gelatin hydrogel with $\mathrm{pH}$ response properties," Journal of Nanomaterials, vol. 2017, Article ID 5139609, 7 pages, 2017.

[16] M. Molina, M. Asadian-Birjand, J. Balach, J. Bergueiro, E. Miceli, and M. Calderon, "Stimuli-responsive nanogel composites and their application in nanomedicine," Chemical Society Reviews, vol. 44, no. 17, pp. 6161-6186, 2015.

[17] A. Sharma, T. Garg, A. Aman et al., "Nanogel—an advanced drug delivery tool: current and future," Artificial Cells, Nanomedicine and Biotechnology, vol. 44, no. 1, pp. 165-177, 2014. 


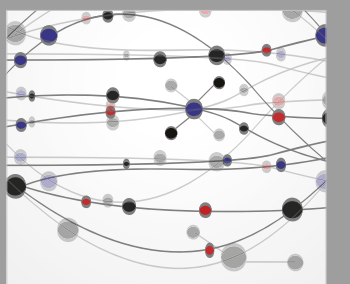

The Scientific World Journal
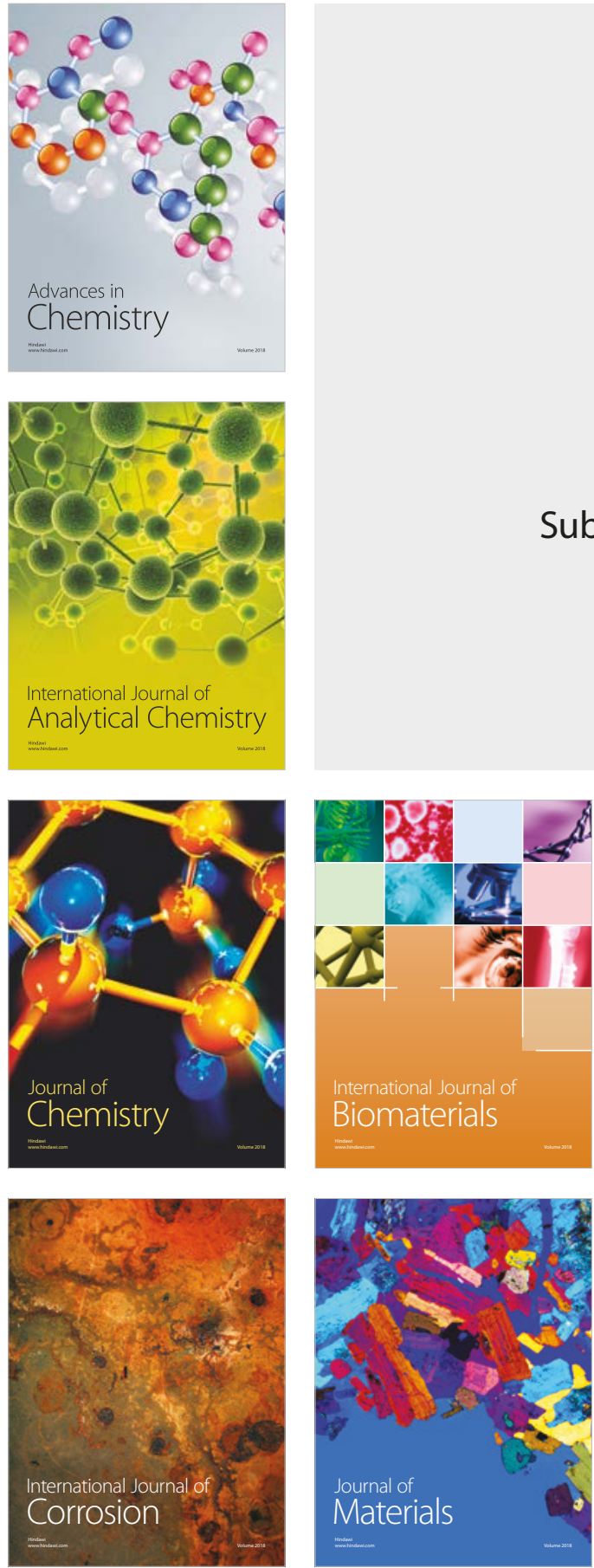

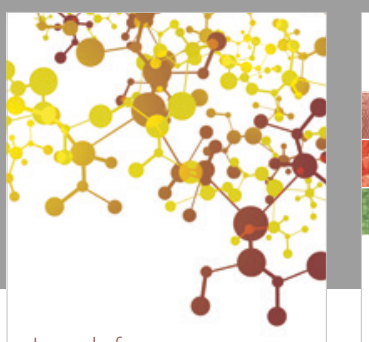

Journal of

Applied Chemistry
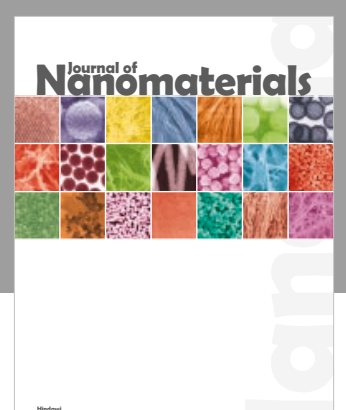

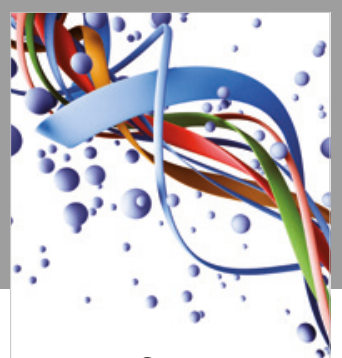

Scientifica

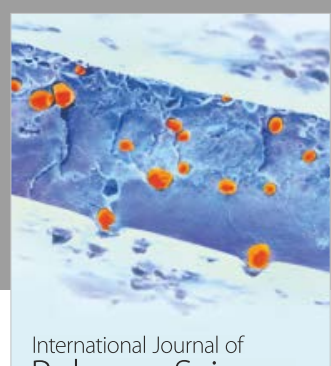

Polymer Science

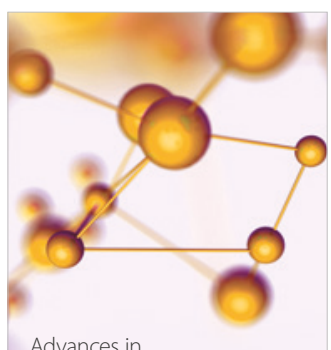

Physical Chemistry
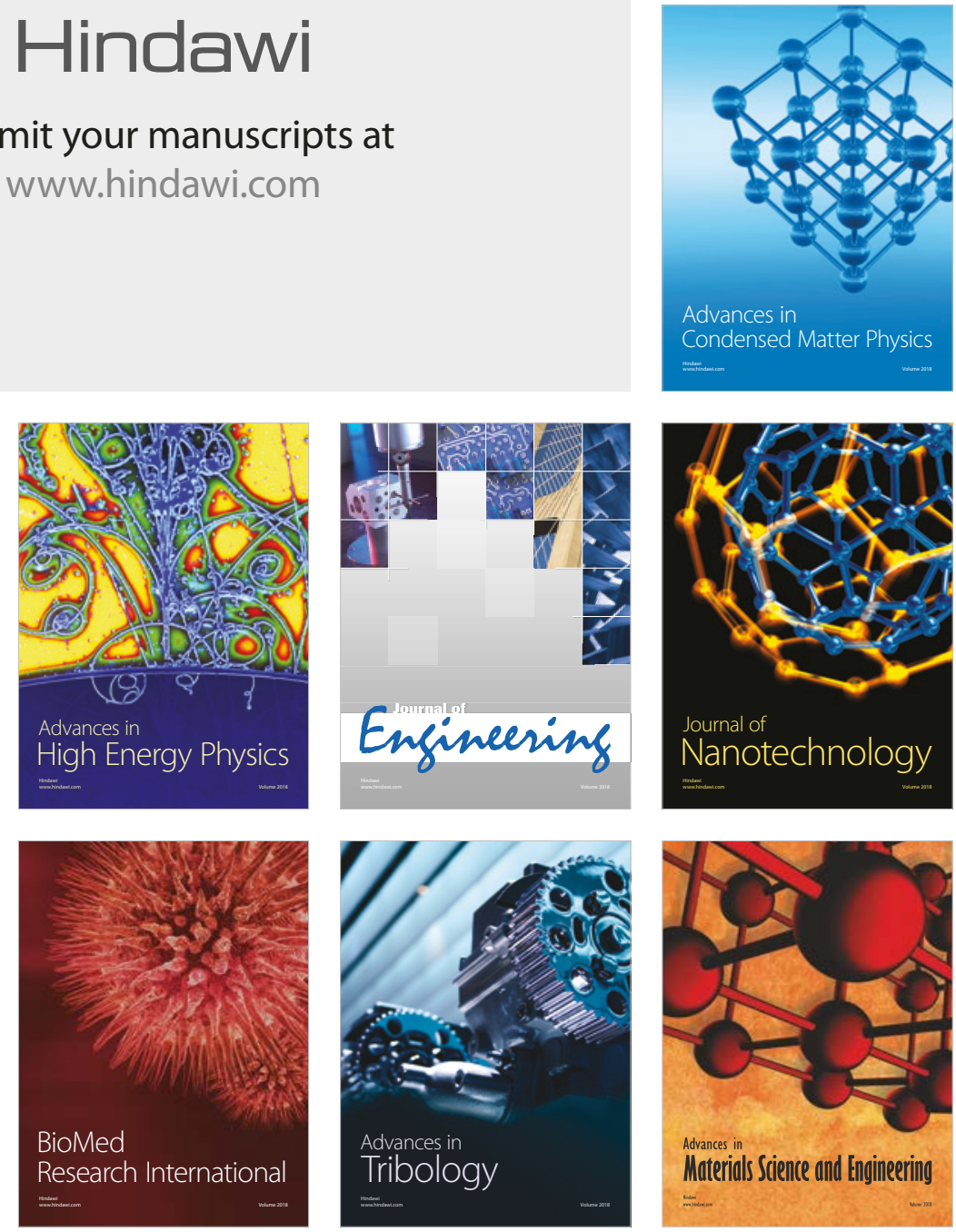